\title{
Optimal Curvature-constrained Paths for General Directional-Cost Functions
}

\author{
Alan J. Chang · Marcus Brazil . \\ J. Hyam Rubinstein · Doreen A. Thomas
}

Received: date / Accepted: date

\begin{abstract}
This paper studies the problem of finding the minimum cost curvature-constrained path between two directed points where the cost at every point along the path depends on the instantaneous direction. This generalises the results obtained by Dubins for curvatureconstrained paths of minimum length, commonly referred to as Dubins paths. We show that there always exists a path of the form $\mathcal{C S C S C}$ or a degeneracy which is optimal, where $\mathcal{C}$ represents an arc of maximum curvature, and $\mathcal{S}$ represents a straight line. This result is also extended to the case where there is not only a directional-cost, but the cost of curved sections are scaled up by a factor $w^{C} \geq 1$. The results obtained can be applied to optimising the development of underground mine networks, where the paths need to satisfy a curvature constraint, the cost of development of the tunnel depends on the direction due to the geological characteristics of the ground, and curved sections may incur more cost due to additional support and ventilation.
\end{abstract}

Keywords Curvature constraint $\cdot$ Dubins paths $\cdot$ Path optimization · Directional cost

Alan J. Chang

Department of Mechanical Engineering, The University of Melbourne, Victoria 3010, Australia

Tel.: +61-3-83446661

Fax: $+61-3-83444290$

E-mail: a.chang5@pgrad.unimelb.edu.au

Marcus Brazil

Department of Electrical and Electronic Engineering, The University of Melbourne, Victoria 3010, Australia

Tel.: +61-3-83443829

Fax: $+61-3-83446678$

E-mail: brazil@unimelb.edu.au

J. Hyam Rubinstein

Department of Mathematics and Statistics, The University of Melbourne, Victoria 3010, Australia

Tel.: +61-3-83445548

Fax: +61-3-83444599

E-mail: rubin@ms.unimelb.edu.au

Doreen A. Thomas

Department of Mechanical Engineering, The University of Melbourne, Victoria 3010, Australia

Tel.: +61-3-83446663

Fax: +61-3-83444290

E-mail: doreen.thomas@unimelb.edu.au 


\section{Introduction}

The problem studied in this paper is that of finding a minimum cost curvature-constrained path between two directed points in $\mathbb{R}^{2}$ where the cost at every point along the path depends on the instantaneous direction. The principal motivation for studying this problem stems from the problem of designing a minimum cost underground transport tunnel, such as a haulage tunnel in an underground mine, in the presence of directional faulting in the ground. This fracturing of the surrounding rock means that the per meter cost of building the tunnel depends of the direction of development, due largely to variability in the amount of support required [6]. Other natural applications are to minimum cost navigation paths for ships or planes, where the presence of currents or winds leads to different costs for different directions [4]. The resulting problem is a natural generalisation of the Dubins problem [5] and is mathematically interesting in its own right.

If the cost of the path is independent of the direction, the problem studied would reduce to the problem of finding the shortest curvature-constrained path between two directed points. This problem was first studied by Dubins [5] who found there were only a few possible forms for the shortest paths. The forms of optimal paths were shown to be the same as Dubins paths in [3] and [4] where the cost is direction-dependent but the reciprocal of the cost function is strictly polarly convex i.e. corresponds to the boundary of a strictly convex set in $\mathbb{R}^{2}$ when plotted in polar coordinates. This reciprocal of the directional-cost is referred to as the velocity function. It was also shown that if the velocity function is weakly polarly convex, there exists a Dubins path which is optimal. This paper further generalises the problem by establishing the forms of optimal paths when the velocity function is polarly non-convex.

The main result in this paper is that there exists a curvature-constrained path of the form $\mathcal{C S C S C}$ which is an optimal path, for any continuous piecewise $C^{2} 2 \pi$-periodic positive directional cost function, where $\mathcal{C}$ represents an arc of maximum curvature, and $\mathcal{S}$ represents a straight line. This is proved for a slightly restricted set of cost functions (whose reciprocals are strictly polarly non-convex) in Theorem 2 , and then extended to the more general statement above in Corollary 3. The strategy employed in proving this result is to make use of the CS-path deformations introduced in Section 3. The basic idea is that if the path has sufficient flexibility, we are able to deform it into a different curvature-constrained path between the same directed points. If the deformation can be performed in both directions (extension and contraction), the effect on the total cost of the path is either strictly negative in one of the two directions, or the deformation can be performed until the path reduces to a simpler form which is no longer flexible enough to reduce further. For some cases, we also need to consider deformations that can only be performed in the extension direction.

Finally, we consider the case where we also impose a scaling factor $w^{C} \geq 1$ on the cost of curved sections, and show that the main result still holds with very simple modifications to the original arguments. This particular extension is motivated by the underground mine development application, where curved sections may incur a greater cost in terms of development as well as increased ventilation costs due to shock losses around bends [7].

Software being developed for optimising underground mine decline design such as in [2] does not take into account geological characteristics of development cost. The main result in this paper will form the basis for future work aimed at designing an efficient algorithm which can then be implemented into the existing software so that the model reflects the true ground conditions more realistically. 


\section{Background}

We first define what we mean by curvature-constrained paths and directional cost as formulated in [3].

\subsection{Curvature-constrained paths}

In the following, $a, b \in \mathbb{R}^{2}$ and $p, q \in \mathbb{R}^{2} \times \mathbb{R} / 2 \pi$.

A path from $a$ to $b$ is a directed piecewise $C^{1}$ curve from $a$ to $b$. We parametrise our paths by arc-length, $E:\left[0, t_{f}\right] \rightarrow \mathbb{R}^{2}$ with $E(0)=a$ and $E\left(t_{f}\right)=b$. The direction $\alpha \in \mathbb{R} / 2 \pi$ at a differentiable point of a path $E(t)$ is given by the polar angle of the tangent at the point in the direction of increasing $t$. At the endpoints, the directions are taken as the respective limits of $\alpha$.

Given a minimum turning circle radius $R>0$, a curvature-constrained path between two directed points $p, q$ is a $C^{1}$ path that is piecewise $C^{2}$, where the absolute curvature everywhere along the path is bounded above by $1 / R$, and the given directions at the start and end points coincide with the directions of $p$ and $q$ respectively. Without loss of generality, we assume the minimum turning circle radius $R=1$ in this entire paper. Let $\mathcal{C}$ be a label denoting a continuous subset of the unit circle. Let $\mathcal{S}$ be a label denoting a straight line segment.

A CS-path is a curvature-constrained path $E:\left[0, t_{f}\right] \rightarrow \mathbb{R}^{2}$ such that there exist $t_{0}, \cdots, t_{n}$ such that $t_{0}=0$ and $t_{n}=t_{f}, t_{i-1}<t_{i}$ for $i=1, \cdots, n$, where $E$ is not twice differentiable at $t_{i}$ for $i=1, \cdots, n$, and each subpath $E_{i}:\left[t_{i-1}, t_{i}\right] \rightarrow \mathbb{R}^{2}$ is either a $\mathcal{C}$ arc or $\mathcal{S}$ segment. The form of such a CS-path is then the sequence of $\mathcal{C}$ and $\mathcal{S}$ labels in ascending order of $i$. The sense of a $\mathcal{C}$ can be further specified using the labels $\mathcal{L}$ and $\mathcal{R}$ for left-turning and right-turning arcs respectively. In the form of a CS-path, any consecutive $\mathcal{C}$ arcs must be of opposite sense due to the condition that the CS-path is differentiable but not twice differentiable at the point in between two consecutive labels. This condition also implies that there will never be consecutive $\mathcal{S}$ labels in the form of a CS-path.

Dubins [5] first studied the problem of finding the shortest curvature-constrained path between any two given directed points in $\mathbb{R}^{2}$ and proved the following theorem.

Theorem 1 Given any two directed points $p, q \in \mathbb{R}^{2} \times \mathbb{R} / 2 \pi$ and a prescribed minimum radius of curvature $R$, the shortest curvature-constrained path is a CS-path with one of the following forms.

1. $\mathcal{C S C}$

2. $\overline{\mathcal{C}} \mathcal{C}$

3. any degeneracies of the two forms

where $\overline{\mathcal{C}}$ denotes a $\mathcal{C}$ arc of length greater than $\pi R$

CS-paths of the forms described in Theorem 1 will be referred to as Dubins paths. Note that there are at most 6 distinct Dubins paths for any pair $p, q$ because of the different sense of the $\mathcal{C}$ arcs, but they do not all necessarily exist and degeneracies may occur.

Theorem 1 was later proved again by Boissonnat et al. [1] by formulating it as a control problem and applying Pontryagin's Minimum Principle. A more detailed treatment of this approach can be found in [8]. 


\subsection{Directional Cost}

Let $\mathbb{R}_{+}$denote the set of all strictly positive real numbers.

A directional-cost function is a continuous, piecewise $C^{2}$ function $c: \mathbb{R} / 2 \pi \rightarrow \mathbb{R}_{+}$. The corresponding velocity function $v: \mathbb{R} / 2 \pi \rightarrow \mathbb{R}_{+}$is given by $v(\alpha)=1 / c(\alpha)$, which is also continuous and piecewise $C^{2}$. The interpretation of the velocity function in the context of directional-cost is a measure of the distance that can be travelled in the direction $\alpha$ at the cost of one unit.

Given a velocity function $v$, the velocity set $V$ is the subset of $\mathbb{R}^{2}$ enclosed by the polar plot of $v$ represented in polar coordinates by

$$
V=\{e=(\varepsilon(\alpha), \alpha): \varepsilon(\alpha) \in[0, v(\alpha)] \forall \alpha \in \mathbb{R} / 2 \pi\}
$$

Let $K=v^{2}+2 v^{\prime 2}-v v^{\prime \prime}$ which is defined for almost all $\alpha$ since $v$ is piecewise $C^{2}$. The sign of $K$ represents the sign of the curvature of the polar function $v$. In particular, if $K=0$ on an interval $\left[\alpha_{1}, \alpha_{2}\right]$, where $\alpha_{1} \neq \alpha_{2}$, this corresponds to $v$ being a straight line in polar coordinates from $\left(v\left(\alpha_{1}\right), \alpha_{1}\right)$ to $\left(v\left(\alpha_{2}\right), \alpha_{2}\right)$. Note that the definition of $K$ here is the negative of the definition of $K$ in [3]. The definition has been changed so that the sign of $K$ represents the sign of the curvature for $v$ in accordance with the usual right-hand rule for sign of curvature in the plane.

Definition 1 A velocity function $v$ is strict if there are no intervals where $K=v^{2}+2 v^{\prime 2}-$ $v v^{\prime \prime}=0 . v$ is weak if it is not strict.

Given a path $E$ and a directional-cost function $c$,

- the length of $E, L(E)$, is given by $L(E)=\int_{E} \mathrm{~d} s$.

- $E$ is degenerate if it has zero length.

- the cost of $E, T(E)$, is given by $T(E)=\int_{E} c(\alpha) \mathrm{d} s$

Let $\mathbb{P}_{p q}$ denote the set of all curvature-constrained paths between two directed points $p$ and $q$. An optimal path from $p$ to $q$ is a path $E \in\left\{P \in \mathbb{P}_{p q}: T(P) \leq T(Q) \forall Q \in \mathbb{P}_{p q}\right\}$.

Lemma 1 If the velocity function is strict, every optimal curvature-constrained path is a CS-path.

Lemma 1 has been proven in [3] and [4]. The proof involves formulating the problem in an optimal control framework and applying Pontryagin's Minimum Principle, based on the method used in [8], but extending the idea to incorporate a direction-dependent velocity. The reason why the velocity function must be strict is to prevent the case where there can exist an optimal path which is not a CS-path. This result will be used in Section 4.

\section{CS-Path Deformations}

In this section, we will introduce deformations to CS-paths which will be used for proving the results in Section 4. 
3.1 Reversible and irreversible deformations

Let $E$ be a CS-path from $p$ to $q$. Let $r$ be a point along a $\mathcal{C}$ subpath of $E$, with direction $\alpha_{r}$. We can form a new CS-path $E^{\prime}$ by fixing the path from $p$ to $r$, introducing an $\mathcal{S}$ subpath of length $\delta$ in the direction $\alpha_{r}$, and then translating the remainder of the original path (from $r$ to $q$ ) by $\delta$ in direction $\alpha_{r}$ so that the new path ends up at $q^{\prime}$ (see Figure 1). We refer to this action as an irreversible extension. Clearly, the resulting position of $q^{\prime}$ is a distance of $\delta$ away from $q$, in the direction $\alpha_{r}$.

On the other hand, if $r$ is a point on an $\mathcal{S}$ subpath of $E$, then this action is equivalent to extending the $\mathcal{S}$ subpath as shown in Figure 2. Furthermore, $r$ being a point on an $\mathcal{S}$ subpath means that we could contract the length of that $\mathcal{S}$ subpath. This can be seen in the same figure as the extension, by treating $q^{\prime}$ as the original endpoint and $q$ as the endpoint after deformation. Since this action can be performed as both an extension and a contraction, we will refer to this as a reversible deformation. Note that the contraction has exactly the opposite effect on $q$ as the extension.

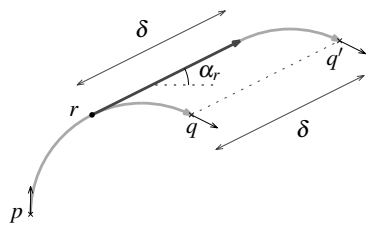

Fig. 1 Irreversible extension at a point $r$ along a $C$ subpath by introducing an $\mathcal{S}$ subpath of length $\delta$. The resulting CS-path ends at $q^{\prime}$ which is a distance $\delta$ away from $q$, in the direction $\alpha_{r}$.

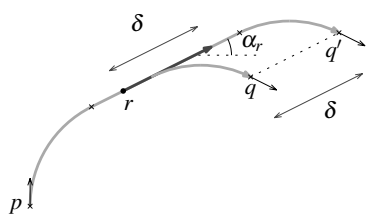

Fig. 2 Reversible deformation at a point $r$ along an $\mathcal{S}$ subpath. The extension can be seen by considering the path ending at $q$ to be the original path, while the contraction can be seen by considering the path ending at $q^{\prime}$ as being the original path.

We also consider reversible deformations taking place at inflection points in $\mathcal{C C}$ subpaths. Let $r$ be an inflection point in an $\mathcal{R} \mathcal{L}$ subpath as shown in Figure 3. We can form a new CS-path by fixing the path from $p$ to $r$, introducing an $\mathcal{L}$ of length $2 \gamma$ starting with direction $\alpha_{r}$, followed by an $\mathcal{R}$ of length $2 \gamma$ starting with direction $\alpha_{r}+2 \gamma$, letting the new path end up at $q^{\prime}$ (see Figure 3(b)). Clearly, the resulting position of $q^{\prime}$ is a distance of $4 \sin \gamma$ away from $q$, in the direction $\alpha_{r}+\gamma$. We can perform an analogous contraction by shortening the existing $\mathcal{L} \mathcal{R}$ subpath starting from $r$, until both arcs are $2 \gamma$ shorter. The contraction to the same original path is shown separately in Figure 3(c) for clarity. Observe that the effect on the resulting position of $q^{\prime}$ is equal and opposite in these two deformations. By considering the mirror images of these figures, we get the corresponding actions for the situation where, instead, $r$ is an inflection point in an $\mathcal{L R}$ subpath.

Observe that it is the properties of the path at $r$, rather than the location of $r$, that are relevant when considering the effect on the resulting position $q^{\prime}$. The types of deformations that can be performed only depend on $\alpha_{r}, \delta$ (or $\gamma$ ) and whether $r$ was a point on a $\mathcal{C}, \mathcal{S}$ or the inflection point of a $\mathcal{C C}$. Furthermore, there are only a finite number of straight line segments and inflection points on a given CS-path. Hence, there are only a finite number of essentially different choices for reversible deformations, whereas irreversible extensions can be performed in any direction $\alpha$, provided there exists a point $r$ which has direction $\alpha$. Note that the size of these deformations were greatly exaggerated in Figures 1 - 3 for clarity. We now formalise the previous discussion as follows. 


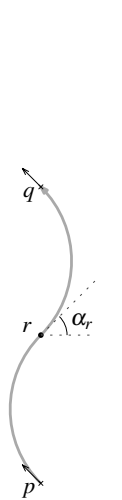

(a) Original path

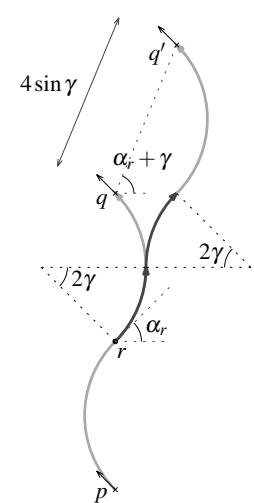

(b) Extended path

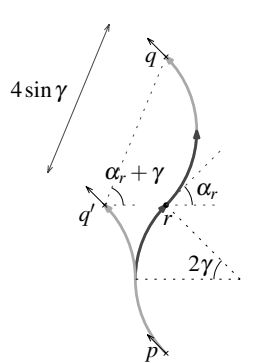

(c) Contracted path

Fig. 3 The original path from $p$ to $q$ with an inflection point at $r$ is shown in (a). This path is deformed reversibly as illustrated in (b) and (c). In (b), two arcs of length $2 \gamma$ are added at $r$, while in (c), the existing two arcs are shortened by $2 \gamma$ each, around $r$.

\subsection{Reversible directions}

The degree $d$ of a CS-path is the number of straight line segments and inflection points between two consecutive arcs in the path. Each of these represents a distinct interval or point at which a reversible CS-path deformation can be performed. A CS-path is reducible if it can be deformed to a CS-path of strictly lower degree with cost no greater than the original CS-path.

Lemma 2 The degree $d$ can be expressed as $d=C+S_{\text {end }}-1$ where $C$ is the number of $\mathcal{C}$ labels in the CS-path and $S_{\text {end }}$ is the number of $\mathcal{S}$ labels in the first and last labels of the CS-path.

Proof Suppose the CS-path begins and ends with label $\mathcal{C}$. Observe that in between every consecutive $\mathcal{C}$ label in the CS-path, there is exactly one of either an inflection point, or a straight line segment. Hence, in this case, $d=C-1$. Now, by simply considering any CSpath, it is clear that having a first label or last label as $\mathcal{S}$ increases the number of straights by one each, and hence $d=C+S_{\text {end }}-1$.

Note that if the CS-path is composed of only an $\mathcal{S}$, then both its first and last labels are $\mathcal{S}$, and hence $S_{\text {end }}=2$.

Definition 2 Given a CS-path with degree $d$, let $\alpha_{i}$ for $i=1, \cdots, d$ denote the directions of the straights and inflection points in the order traversed by the path. We define $A_{i}=\left(\alpha_{i}, \rho_{i}\right)$ for $i=1, \cdots, d$ to be reversible directions, where

$$
\rho_{i}=\left\{\begin{array}{cl}
-1 & \text { if it corresponds to the inflection point in an } \mathcal{L} \mathcal{R} \text { subpath } \\
0 & \text { if it corresponds to a straight } \mathcal{S} \text { subpath } \\
1 & \text { if it corresponds to the inflection point in an } \mathcal{R} \mathcal{L} \text { subpath }
\end{array}\right.
$$

Given a CS-path, we denote by $\mathbf{C}_{k}$ a particular subpath of the form $\mathcal{C}$ where $k \in \mathbb{Z}_{+}$is simply an index to distinguish different subpaths. $\mathbf{L}_{k}, \mathbf{R}_{k}, \mathbf{S}_{k}$ are defined similarly.

Consider a CS-path $E$ from $p$ to $q$ which has a reversible direction $A_{i}=\left(\alpha_{i}, \rho_{i}\right)$. We can create a new CS-path $E^{\prime}$ which starts from $p$ and ends at $q^{\prime}$ by performing a number 
of small reversible deformations. In the following, $\gamma_{i}$ is a parameter controlling the size of a reversible deformation. We will consistently use the following notation in the paper for brevity.

$-\delta_{i}=4 \sin \gamma_{i}$

$-\theta_{i}=\alpha_{i}+\rho_{i}\left|\gamma_{i}\right|$

$-\xi_{\mathbf{i}}=\left(\cos \theta_{i}, \sin \theta_{i}\right)$.

Given a reversible direction $\left(\alpha_{i}, \rho_{i}\right)$, there exists $\varepsilon>0$ such that for $\gamma_{i} \in(-\varepsilon, \varepsilon)$ we can find a CS-path from $p$ to the directed point $q+\delta_{i}\left(\xi_{\mathbf{i}}, 0\right)$. Let $c(\alpha)$ denote the given directional-cost function, and recall the definition of the cost of a path in Section 2.2. If $\rho_{i}=0$, the resulting change in cost is $\delta_{i} c\left(\alpha_{i}\right)$, otherwise it is $2 \int_{0}^{2 \gamma_{i}} c\left(\alpha_{i}+2 \rho_{i}|\phi|\right) \mathrm{d} \phi$. Note that $\gamma_{i}>0$ (extension) leads to an increase in cost, while $\gamma_{i}<0$ (contraction) leads to a decrease in cost.

Given a point on a path $E$ with a corresponding reversible direction $A_{i}$, applying a deformation at that point for which $\gamma_{i}>0$ will be called extending along $A_{i}$ while applying a deformation with $\gamma_{i}<0$ will be called contracting along $A_{i}$. Note that in Section 3.1, $\delta$ and $\gamma$ were introduced as positive quantities. We now let $\delta_{i}<0$ and $\gamma_{i}<0$ represent the corresponding contractions of length $\left|\delta_{i}\right|$ and $\left|\gamma_{i}\right|$, but continue to label distances and angles on diagrams with $\delta_{i}$ and $\gamma_{i}$ even when they take negative values, since it is now clear what they represent.

A direction plot of a CS-path is a polar plot of all the directions traversed along that path, plotted at increasing radii for different $\mathcal{C}$ arcs in the order traversed, with radial line segments representing $\mathcal{S}$ segments. This contains all the information required to compute the total cost of that path, provided the lengths of all $\mathcal{S}$ segments are separately specified.

The example depicted in Figure 4 demonstrates the purpose of considering reversible directions. The reversibility of the deformations is explicitly illustrated in Figures 4(b) and 4(c). The direction plot of the $\mathcal{C} \mathcal{S C C}$ path from Figure 4(a) shown in Figure 5, also shows the direction plot of the contracted path from Figure 4(c) by considering only the lighter grey arrows. In Figure $6, \gamma_{1}$ and $\gamma_{3}$ have been exaggerated to clearly illustrate the geometric meaning of $\gamma_{i}$ and $\theta_{i}$. In this example, $\gamma_{1}, \gamma_{2}$ and $\gamma_{3}$ are chosen such that the net result of the deformation results in $q^{\prime}=q$. This will be one of the main strategies employed in applying reversible directions to formulate arguments in the next section.

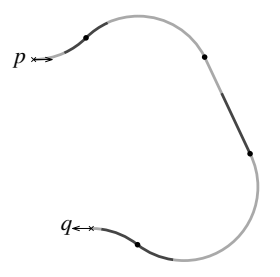

(a) Original $\mathcal{C C S C C}$ path

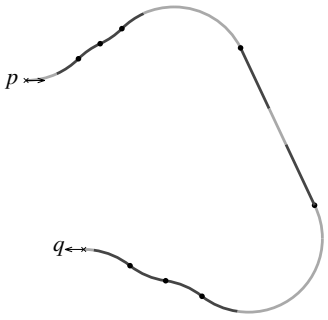

(b) Extending to a $\mathcal{C} C \mathcal{C S C C C C}$ path

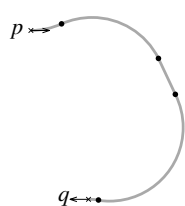

(c) Contracting to a shorter $\mathcal{C} \mathcal{S C C}$ path

Fig. 4 Example of reversible deformations performed on a $\mathcal{C C S C C}$ path from $p$ to $q$ while keeping the endpoint as $q$. The 3 darker grey portions of the original path in (a) are replicated in (b) and removed in (c), as a result of the respective extending and contracting along the 3 reversible directions 


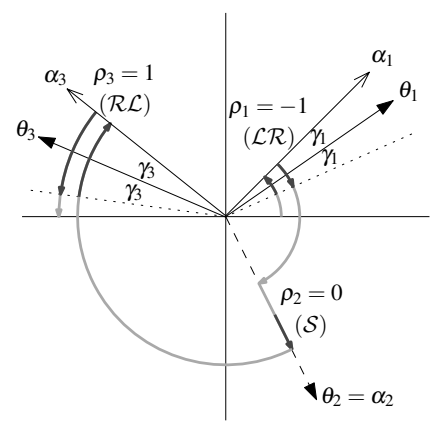

Fig. 5 Direction plot of $\mathcal{C C S C C}$ path from Figure $4($ a)

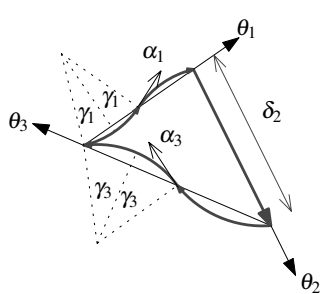

Fig. 6 Schematic diagram of the darker grey arcs and straights from Figure 4(a) connected sequentially

For brevity, we say that a set of directions spans $\mathbb{R}^{2}$ if their corresponding unit vectors span $\mathbb{R}^{2}$. Likewise, a set of directions positively span $\mathbb{R}^{2}$ if their corresponding unit vectors positively span $\mathbb{R}^{2}$. In order to identify what the appropriate combination of CS-path deformations to consider are, we need to consider whether a set of reversible directions positively spans $\mathbb{R}^{2}$. For most cases, a set of reversible directions $A_{1}, \cdots, A_{n}$ positively spans $\mathbb{R}^{2}$ if and only if $\alpha_{1}, \cdots, \alpha_{n}$ positively spans $\mathbb{R}^{2}$. However, this is not always the case as the extension or contraction of a reversible direction $A_{i}$, where $\rho_{i} \neq 0$, is not exactly in the direction $\alpha_{i}$ even for an arbitrarily small deformation. This is why we need to formally define positive spanning for reversible directions as follows:

Definition 3 A set of reversible directions $A_{1}, \cdots, A_{n}$

- positively span $\mathbb{R}^{2}$ if there exists $\varepsilon>0$ such that for all $\eta_{1}, \cdots, \eta_{n} \in(0, \varepsilon), \alpha_{1}+\rho_{1} \eta_{1}, \cdots, \alpha_{n}+$ $\rho_{n} \eta_{n}$ positively span $\mathbb{R}^{2}$.

- lie in an open half-space of $\mathbb{R}^{2}$ if there exists $\varepsilon>0$ such that for all $\eta_{1}, \cdots, \eta_{n} \in(0, \varepsilon)$, $\alpha_{1}+\rho_{1} \eta_{1}, \cdots, \alpha_{n}+\rho_{n} \eta_{n}$ lie in an open half-space of $\mathbb{R}^{2}$.

Clearly, any set of reversible directions satisfies at most one of these two properties. A pair of reversible directions $A_{i}, A_{j}(i \neq j)$ are

- repeated if $A_{i}=A_{j}$ (i.e. $\alpha_{i}=\alpha_{j}$ and $\rho_{i}=\rho_{j}$ ).

- opposite if $\alpha_{i}=\alpha_{j}+\pi$ and $\rho_{i}=\rho_{j}$.

In a set of reversible directions, the reversible directions are said to be distinct if there are no repeated reversible directions in the set.

It is clear by this definition that if each pair of directions $\alpha_{j}, \alpha_{k}$ are linearly independent for $j, k=1, \cdots, n$ and $j \neq k$, then $A_{1}, \cdots, A_{n}$ positively span $\mathbb{R}^{2}$ iff $\alpha_{1}, \cdots, \alpha_{n}$ positively span $\mathbb{R}^{2}$ and likewise for lying in an open half-space of $\mathbb{R}^{2}$. However, the following example illustrates the difference when a pair of directions are linearly dependent.

Example 1 Consider a CS-path with reversible directions $A_{i}=\left(\alpha_{i}, \rho_{i}\right)$ for $i=1,2,3$, where:

- $A_{1}=(\pi, 0)$

$-A_{2}=(\pi / 2,0)$

$-\alpha_{3}=0$.

It is clear that $\alpha_{1}, \alpha_{2}, \alpha_{3}$ do not positively span $\mathbb{R}^{2}$ nor lie in an open half-space of $\mathbb{R}^{2}$ and $\alpha_{1}, \alpha_{3}$ are linearly dependent. However, by Definition 3, 
- if $\rho_{3}=-1, A_{1}, A_{2}, A_{3}$ positively span $\mathbb{R}^{2}$.

- if $\rho_{3}=0, A_{1}$ and $A_{3}$ are opposite.

- if $\rho_{3}=1, A_{1}, A_{2}, A_{3}$ lie in an open half-space of $\mathbb{R}^{2}$.

This example shows that for some cases, knowing $\alpha_{1}, \cdots, \alpha_{n}$ alone without information of $\rho_{1}, \cdots, \rho_{n}$ is insufficient to tell us exactly whether or not $A_{1}, \cdots, A_{n}$ positively span $\mathbb{R}^{2}$, lie in an open half-space of $\mathbb{R}^{2}$, or contain a pair of repeated or opposite reversible directions. We will show later in Section 4 that knowing which of these properties hold will be important for determining what combination of deformations we should consider in order to show that the given path is not optimal, or can be reduced to a simpler form.

In the example illustrated by Figure 4, there exist $\gamma_{1}, \gamma_{2}, \gamma_{3}$ such that the deformed paths were distinct from original path but $q^{\prime}=q$. We need to show that this is true given any 3 reversible directions, since our arguments rely on the existence of such values. Furthermore, we need to show that we can construct this new path arbitrarily close to the original path.

Lemma 3 Let $A_{i}, A_{j}, A_{k}$ be three reversible directions of a CS-path from $p$ to $q$. Given any $\varepsilon>0$, there exist $\gamma_{i}, \gamma_{j}, \gamma_{k} \in(-\varepsilon, \varepsilon)$ such that the resulting path is distinct from the original but $q^{\prime}=q$.

Proof Recall that $\delta_{i}=4 \sin \gamma_{i}, \theta_{i}=\alpha_{i}+\rho_{i}\left|\gamma_{i}\right|$ and $\xi_{\mathbf{i}}=\left(\cos \theta_{i}, \sin \theta_{i}\right)$.

In order for the resulting path to terminate at $q, \gamma_{i}, \gamma_{j}, \gamma_{k}$ must satisfy the following condition.

$$
\delta_{i} \xi_{\mathbf{i}}+\delta_{j} \xi_{\mathbf{j}}+\delta_{k} \xi_{\mathbf{k}}=0
$$

If there exists a pair of repeated reversible directions, without loss of generality let them be $A_{i}$ and $A_{j}$. Since there exists $\varepsilon_{1}>0$ such that the deformations are valid for $\gamma_{i}, \gamma_{j} \in\left(-\varepsilon_{1}, \varepsilon_{1}\right)$, we can simply choose $\gamma_{i}=\left(\min \left\{\varepsilon_{1}, \varepsilon\right\}\right) / 2, \gamma_{j}=-\left(\min \left\{\varepsilon_{1}, \varepsilon\right\}\right) / 2, \gamma_{k}=0$ to satisfy (1).

Similarly, if there are a pair of opposite reversible directions, (1) is easily satisfied.

Suppose that the directions $\alpha_{i}, \alpha_{j}, \alpha_{k}$ span $\mathbb{R}^{2}$. Without loss of generality, let $\alpha_{i}$ and $\alpha_{j}$ $\operatorname{span} \mathbb{R}^{2}$.

Consider $F: \mathbb{R}^{2} \rightarrow \mathbb{R}^{2}$ defined as follows.

$$
F\left(\gamma_{i}, \gamma_{j}\right)=\delta_{i} \xi_{\mathbf{i}}+\delta_{j} \xi_{\mathbf{j}}
$$

The Jacobian of $F$ can be simplified as follows.

$$
J\left(\gamma_{i}, \gamma_{j}\right)=4\left[\begin{array}{ll}
\cos \left(\alpha_{i}+2 \rho_{i}\left|\gamma_{i}\right|\right) & \cos \left(\alpha_{j}+2 \rho_{j}\left|\gamma_{j}\right|\right) \\
\sin \left(\alpha_{i}+2 \rho_{i}\left|\gamma_{i}\right|\right) & \sin \left(\alpha_{j}+2 \rho_{j}\left|\gamma_{j}\right|\right)
\end{array}\right]
$$

The determinant of $J$ evaluated at the origin is given by

$$
\operatorname{det}(J)=16 \sin \left(\alpha_{j}-\alpha_{i}\right)
$$

which is nonzero since $\alpha_{i}$ and $\alpha_{j}$ span $\mathbb{R}^{2}$. Since $J$ is continuous at the origin, by the Inverse Function Theorem, there exists $\varepsilon_{2}>0$ such that for all $\gamma_{k} \in\left(-\varepsilon_{2}, \varepsilon_{2}\right)$, there exists $\gamma_{i}, \gamma_{j}$ such that (1) is satisfied.

This leaves only one case to consider, which is when $\alpha_{i}, \alpha_{j}, \alpha_{k}$ do not span $\mathbb{R}^{2}$, and there are no repeated reversible directions, nor opposite reversible directions. This is only possible if all three $\rho$ values are different. Without loss of generality, let $\rho_{i}=-1, \rho_{j}=0$, $\rho_{k}=1$. Since there exists $\varepsilon_{3}>0$ such that the deformations are valid for $\gamma_{i}, \gamma_{j}, \gamma_{k} \in\left(-\varepsilon_{3}, \varepsilon_{3}\right)$, 
let $\varepsilon_{4}=\left(\min \left\{\varepsilon_{3}, \varepsilon\right\}\right) / 2$ and choose $\gamma_{i}=\cos \left(\alpha_{i}-\alpha_{j}\right) \varepsilon_{4}, \gamma_{j}=-2 \varepsilon_{4}, \gamma_{k}=\cos \left(\alpha_{k}-\alpha_{j}\right) \varepsilon_{4}$ to satisfy (1).

Corollary 1 Let $A_{i}, A_{j}, A_{k}$ be three reversible directions of a CS-path. If $A_{i}, A_{j}, A_{k}$ positively span $\mathbb{R}^{2}$ then there exist $\gamma_{i}, \gamma_{j}, \gamma_{k}<0$ such that condition (1) is satisfied.

Proof By Definition 3, there exists $\varepsilon \in(0,2 \pi)$ such that $\forall \eta_{i}, \eta_{j}, \eta_{k} \in(0, \varepsilon), \alpha_{i}+\rho_{i} \eta_{i}, \alpha_{j}+$ $\rho_{j} \eta_{j}, \alpha_{k}+\rho_{k} \eta_{k}$ positively span $\mathbb{R}^{2}$. By Lemma 3, there exist $\gamma_{i}, \gamma_{j}, \gamma_{k} \in(-\varepsilon, \varepsilon)$ satisfying (1) while $\left(\gamma_{i}, \gamma_{j}, \gamma_{k}\right) \neq(0,0,0)$. Hence, $\theta_{i}, \theta_{j}, \theta_{k}$ positively span $\mathbb{R}^{2}$.

Since $\left(\gamma_{i}, \gamma_{j}, \gamma_{k}\right) \neq(0,0,0)$ and $\left|\gamma_{i}\right|,\left|\gamma_{j}\right|,\left|\gamma_{k}\right|<2 \pi$, we know $\left(\delta_{i}, \delta_{j}, \delta_{k}\right) \neq(0,0,0)$. Since $\xi_{i}, \xi_{j}, \xi_{k}$ positively span $\mathbb{R}^{2}$ and $\left(\delta_{i}, \delta_{j}, \delta_{k}\right) \neq(0,0,0)$, this means either $\delta_{i}, \delta_{j}, \delta_{k}>0$ or $\delta_{i}, \delta_{j}, \delta_{k}<0$. These two cases correspond to $\gamma_{i}, \gamma_{j}, \gamma_{k}>0$ and $\gamma_{i}, \gamma_{j}, \gamma_{k}<0$ respectively. Hence by choosing $\delta_{i}, \delta_{j}, \delta_{k}<0$, there exist $\gamma_{i}, \gamma_{j}, \gamma_{k}<0$ satisfying condition (1).

Given a set of distinct reversible directions $A_{1}, \cdots, A_{n}$ which lie in an open half-space of $\mathbb{R}^{2}$, a reversible direction $A_{j}$ in this set is an

- interior reversible direction if there exists $\varepsilon>0$ such that $\forall \eta_{1}, \cdots, \eta_{n} \in(0, \varepsilon), \alpha_{j}+$ $\rho_{j} \eta_{j}$ lies strictly inside the cone positively spanned by $\alpha_{1}+\rho_{1} \eta_{1}, \cdots, \alpha_{n}+\rho_{n} \eta_{n}$.

- exterior reversible direction if it is not an interior reversible direction.

Corollary 2 Let $A_{i}, A_{j}, A_{k}$ be three distinct reversible directions which lie in an open halfspace of $\mathbb{R}^{2}$ where $A_{j}$ is the interior reversible direction. There exist $\gamma_{i}, \gamma_{k}<0$ and $\gamma_{j}>0$ such that condition (1) is satisfied.

Proof This result follows in a very similar manner to the proof of Corollary 1, where the sign of $\gamma_{j}$ is now different to $\gamma_{i}, \gamma_{k}$ because $A_{j}$ is the interior reversible direction.

Definition 4 The velocity point of a reversible direction $A_{i}$ is the point in $\mathbb{R}^{2}$ which in polar coordinates is $\left(v\left(\alpha_{i}\right), \alpha_{i}\right)$.

Lemma 4 Let $A_{i}, A_{j}, A_{k}$ be distinct reversible directions which lie in an open half-space of $\mathbb{R}^{2}$ where $A_{j}$ is the interior reversible direction. Let $\rho_{i}, \rho_{j}, \rho_{k}=0$. Let the straight line passing through the velocity points of $A_{i}$ and $A_{k}$ be given in polar coordinates by the function $v_{i}^{k}(\alpha)$. There exist $\delta_{i}, \delta_{k}<0$ and $\delta_{j}>0$ satisfying (1) such that

1. if $v\left(\alpha_{j}\right)>v_{i}^{k}\left(\alpha_{j}\right)$, then $\delta_{i} c\left(\alpha_{i}\right)+\delta_{j} c\left(\alpha_{j}\right)+\delta_{k} c\left(\alpha_{k}\right)<0$

2. if $v\left(\alpha_{j}\right)<v_{i}^{k}\left(\alpha_{j}\right)$, then $\delta_{i} c\left(\alpha_{i}\right)+\delta_{j} c\left(\alpha_{j}\right)+\delta_{k} c\left(\alpha_{k}\right)>0$

3. if $v\left(\alpha_{j}\right)=v_{i}^{k}\left(\alpha_{j}\right)$, then $\delta_{i} c\left(\alpha_{i}\right)+\delta_{j} c\left(\alpha_{j}\right)+\delta_{k} c\left(\alpha_{k}\right)=0$

Proof The existence of $\delta_{i}, \delta_{k}<0$ and $\delta_{j}>0$ satisfying (1) is given by Corollary 2. We start by showing property (3). Recall that since $\rho_{i}, \rho_{j}, \rho_{k}=0, \xi_{i}, \xi_{j}, \xi_{k}$ denote unit vectors in the directions $\alpha_{i}, \alpha_{j}, \alpha_{k}$ respectively. Consider a norm $\|\cdot\|$ on $\mathbb{R}^{2}$ such that its unit sphere contains a straight line from $\left(v\left(\alpha_{i}\right), \alpha_{i}\right)$ to $\left(v\left(\alpha_{k}\right), \alpha_{k}\right)$ in polar coordinates. It is well known (see for example [9]) that in this case, $\left\|\delta_{i} \xi_{i}\right\|+\left\|\delta_{k} \xi_{k}\right\|=\left\|\delta_{j} \xi_{j}\right\|$. Since $\left\|\delta_{i} \xi_{i}\right\|=\delta_{i} / v\left(\alpha_{i}\right)=$ $\delta_{i} c\left(\alpha_{i}\right),\left\|\delta_{j} \xi_{j}\right\|=-\delta_{j} c\left(\alpha_{j}\right)$ and $\left\|\delta_{k} \xi_{k}\right\|=\delta_{k} c\left(\alpha_{k}\right)$, this proves property (3) holds. We can then restate property (3) as $\delta_{i} c\left(\alpha_{i}\right)+\delta_{j} c_{i}^{k}\left(\alpha_{j}\right)+\delta_{k} c\left(\alpha_{k}\right)=0$ where $c_{i}^{k}\left(\alpha_{j}\right)=1 / v_{i}^{k}\left(\alpha_{j}\right)$, from which properties (1) and (2) clearly follow.

We need the following more general result which is not restricted to deformations on straight paths. 
Lemma 5 Let $A_{i}, A_{j}, A_{k}$ be distinct reversible directions which lie in an open half-space of $\mathbb{R}^{2}$ where $A_{j}$ is the interior reversible direction. Let the straight line passing through the velocity points of $A_{i}$ and $A_{k}$ be given in polar coordinates by the function $v_{i}^{k}(\alpha)$. There exist $\gamma_{i}, \gamma_{k}<0$ and $\gamma_{j}>0$ satisfying (1) such that

1. if $v\left(\alpha_{j}\right)>v_{i}^{k}\left(\alpha_{j}\right)$, then $\mathrm{d} T_{i}+\mathrm{d} T_{j}+\mathrm{d} T_{k}<0$

2. if $v\left(\alpha_{j}\right)<v_{i}^{k}\left(\alpha_{j}\right)$, then $\mathrm{d} T_{i}+\mathrm{d} T_{j}+\mathrm{d} T_{k}>0$

3. if $v\left(\alpha_{j}\right)=v_{i}^{k}\left(\alpha_{j}\right)$, and if $v$ is strict, and exactly one of $\rho_{i}, \rho_{j}, \rho_{k}$ is nonzero, then $\mathrm{d} T_{i}+$ $\mathrm{d} T_{j}+\mathrm{d} T_{k} \neq 0$

where $\mathrm{d} T_{i}<0, \mathrm{~d} T_{j}>0, \mathrm{~d} T_{k}<0$ are the respective changes in cost resulting from $\gamma_{i}, \gamma_{j}, \gamma_{k}$ depending on $\rho_{i}, \rho_{j}, \rho_{k}$.

Proof The existence of $\gamma_{i}, \gamma_{k}<0$ and $\gamma_{j}>0$ satisfying (1) is given by Corollary 2. Properties (1), (2) follow by applying Lemma 3 with sufficiently small $\varepsilon$ so that the results hold in a similar manner to (1), (2) in Lemma 4.

Let $v\left(\alpha_{j}\right)=v_{i}^{k}\left(\alpha_{j}\right)$ and exactly one of $\rho_{i}, \rho_{j}, \rho_{k}$ be nonzero. Let $m \in\{i, j, k\}$ denote the reversible direction with nonzero $\rho_{m}$ and without loss of generality, let $\rho_{m}=1$. It is only possible to have $\mathrm{d} T_{j}=\mathrm{d} T_{i}+\mathrm{d} T_{k}$ for arbitrarily small $\gamma_{i}, \gamma_{j}, \gamma_{k} \neq 0$ if there exists a $\varepsilon_{m}>0$ such that for all $\alpha \in\left[\alpha_{m}, \alpha_{m}+\varepsilon_{m}\right], v(\alpha)=v_{i}^{k}(\alpha)$. If $v$ is strict, there exists no such $\varepsilon_{m}>0$, and hence, $\mathrm{d} T_{j} \neq \mathrm{d} T_{i}+\mathrm{d} T_{k}$.

\section{Necessary and Sufficient Path Sets}

From Lemma 1, we know that every optimal path is a CS-path if the velocity function is strict. In this section, we will first use the tools developed in Section 3 to show that for strict velocity functions, all CS-paths with $d \geq 3$ are either non-optimal or reducible to a CS-path with $d \leq 2$ of equal cost. We will establish a sufficient set of CS-paths which always contains an optimal path, and a (larger) necessary set of CS-paths which contains all optimal paths if the velocity function is strict. In other words, it suffices to consider the sufficient set to find an optimal path, while any optimal path necessarily belongs to the necessary set. We then extend the results to weak velocity functions by a simple argument which employs the fact that any weak velocity function is arbitrarily close to a strict velocity function. This proves that the result holds for any velocity function. Finally, we show that this method of proof easily generalises the result to incorporate an additional complication where the cost of curved sections of the path are scaled up by a constant factor $w^{C} \geq 1$.

The following example illustrates the difference between the necessary set and the sufficient set. The path shown in Figure 7(a) is an $\mathcal{S C C S}$ path with $d=3$ belonging only to the necessary set, but is of the same cost as the $\mathcal{C C S}$ path in Figure 7(b) with $d=2$ belonging to both the necessary and sufficient sets.

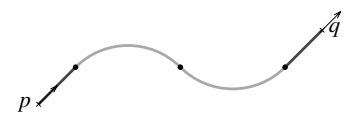

(a) $\mathcal{S C C S}$ path $(d=3)$

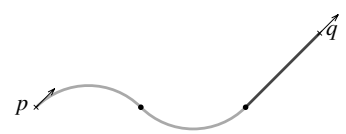

(b) $\mathcal{C C S}$ path $(d=2)$

Fig. 7 Example of a $d=3$ path (a) in the necessary set which has the same cost as a $d=2$ path (b) in the sufficient set. 
We break up all CS-paths with $d \geq 3$ into smaller cases, and prove non-optimality or reducibility to each of these cases individually. To assist the reader in following the flow of the proofs, Tables 1 and 2 have been included which summarise Lemmas 6 - 14 graphically. These also serve to provide a visual check that all cases have been covered, and to keep track of which cases are non-optimal, and which are reducible.

Table 1 Cases covered by Lemmas 6 - 10

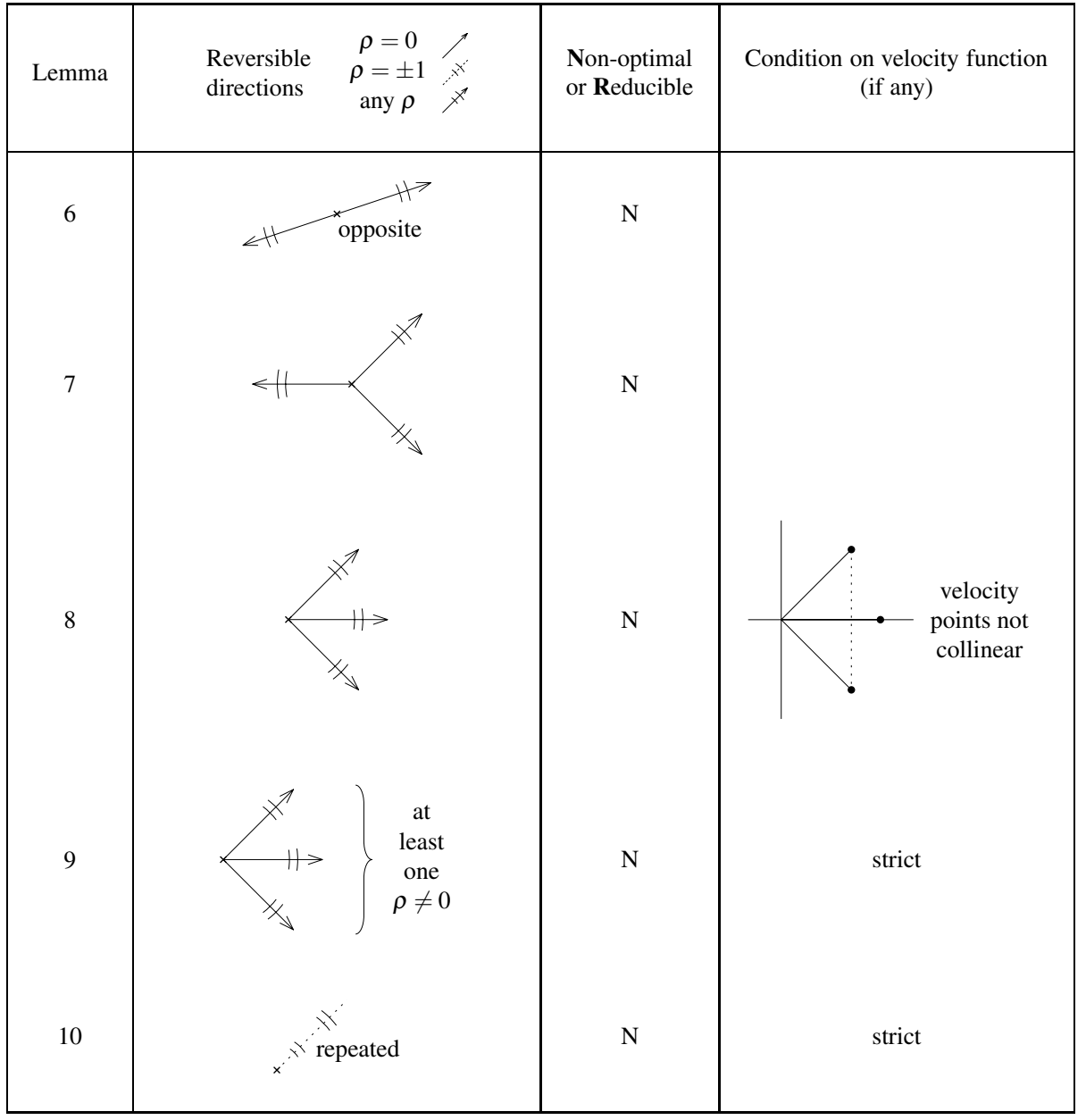

4.1 Non-optimal path forms

Lemma 6 Any CS-path with opposite reversible directions is non-optimal.

Proof Let $E$ be a CS-path from $p$ to $q$ with opposite reversible directions. Contracting along both reversible directions by equal amounts results in a new path $E^{\prime}$ with $q^{\prime}=q$ and $T\left(E^{\prime}\right)<$ $T(E)$. 
Table 2 Cases covered by Lemmas 11 - 15

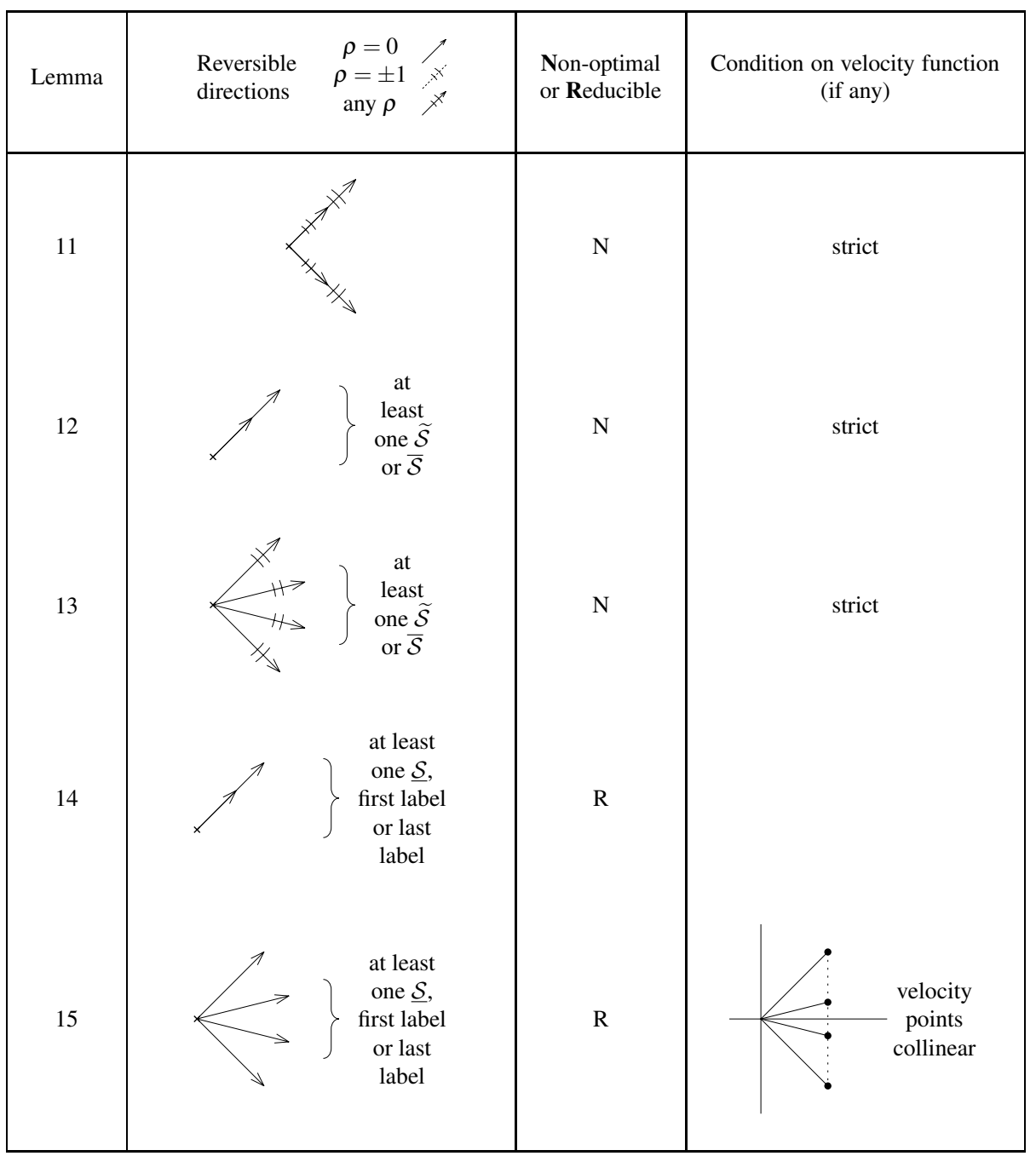

Lemma 7 Any CS-path with 3 distinct reversible directions which positively span $\mathbb{R}^{2}$ is non-optimal.

Proof Let $E$ be a CS-path from $p$ to $q$ with $d \geq 3$ and 3 distinct reversible directions $A_{i}, A_{j}$, $A_{k}$ which positively span $\mathbb{R}^{2}$. By Corollary 1 , there exist $\gamma_{i}, \gamma_{j}, \gamma_{k}<0$ satisfying condition (1). Hence, the new CS-path $E^{\prime}$ formed by contracting along $A_{i}, A_{j}, A_{k}$ by the respective amounts has $q^{\prime}=q$ and $T\left(E^{\prime}\right) \neq T(E)$. By choosing either $E^{\prime}$ or the new path obtained by the reverse deformations, we get a new path with cost strictly less than $T(E)$.

Figure 4(c) illustrates an example of Lemma 7.

Lemma 8 Let $E$ be a CS-path from $p$ to $q$ such that:

- all the reversible directions of $E$ lie in an open half-space of $\mathbb{R}^{2}$; 
- E has at least 3 distinct reversible directions which have non-collinear velocity points.

Then E is non-optimal.

Proof Select 3 distinct reversible directions $A_{i}, A_{j}, A_{k}$ which have velocity points that are not collinear. By properties (1) and (2) of Lemma 5 there exist $\gamma_{i}, \gamma_{j}, \gamma_{k} \neq 0$ satisfying (1). Hence, the new path $E^{\prime}$ formed by the respective deformations along $A_{i}, A_{j}, A_{k}$ has $q^{\prime}=q$ and $T\left(E^{\prime}\right)<T(E)$.

Lemma 9 Let $E$ be a CS-path from $p$ to $q$ such that:

- E has at least 3 distinct reversible directions;

- E has at least 1 reversible direction with $\rho \neq 0$.

If $v$ is strict, then $E$ is non-optimal.

Proof From Lemmas 6 - 8, only the case where all the reversible directions lie in an open half-space of $\mathbb{R}^{2}$, and their velocity points are all collinear, needs to be considered. Select any 3 distinct reversible directions $A_{i}, A_{j}, A_{k}$ such that at least one of $\rho_{i}, \rho_{j}, \rho_{k}$ is nonzero. Without loss of generality, let $A_{j}$ be the interior direction.

If $\rho=0$ for 2 of the 3 reversible directions, then by property (3) of Lemma 5 , there exist $\gamma_{i}, \gamma_{j}, \gamma_{k} \neq 0$ satisfying (1) such that $\mathrm{d} T_{i}+\mathrm{d} T_{j}+\mathrm{d} T_{k} \neq 0$ where $\mathrm{d} T_{i}, \mathrm{~d} T_{j}, \mathrm{~d} T_{k}$ denote the respective changes in cost due to deforming along $A_{i}, A_{j}, A_{k}$. Since this deformation is reversible, it is therefore possible to construct a new path $E^{\prime}$ with $q^{\prime}=q$ so that $T\left(E^{\prime}\right)<$ $T(E)$.

If $\rho_{j} \neq 0$, without loss of generality, let $\rho_{j}=1$. Let the line passing through the collinear velocity points be given in polar coordinates by the function $\bar{v}(\alpha)$. For a sufficiently small $\varepsilon>0$, if there exists a direction $\alpha^{*} \in\left[\alpha_{j}, \alpha_{j}+\varepsilon\right]$ such that $v\left(\alpha^{*}\right)>\bar{v}\left(\alpha^{*}\right)$, introducing a straight in direction $\alpha^{*}$ in either of the two arcs corresponding to $A_{j}$ while contracting along $A_{i}$ and $A_{k}$ will produce a new CS-path $E^{\prime}$ from $p$ to $q$ with $T\left(E^{\prime}\right)<T(E)$. If no such $\alpha^{*}$ exists, then contracting along $A_{j}$ while introducing straights at $\alpha_{i}$ and $\alpha_{k}$, will result in a new CS-path $E^{\prime \prime}$ from $p$ to $q$ with $T\left(E^{\prime \prime}\right) \leq T(E)$ since $v(\alpha) \leq \bar{v}(\alpha) \forall \alpha \in\left(\alpha_{j}, \alpha_{j}+\varepsilon\right]$. However, in this case $T\left(E^{\prime \prime}\right)=T(E)$ would imply that $K(\alpha)=0 \forall \alpha \in\left[\alpha_{j}, \alpha_{j}+\varepsilon\right]$ since this argument holds for arbitrarily small $\gamma_{j}$. Since $v$ is strict, this isn't possible and hence $T\left(E^{\prime \prime}\right)<T(E)$.

This leaves only one case to consider, which is when $\rho_{i}, \rho_{k} \neq 0$, while $\rho_{j}=0$. By a slight modification of Lemma 3, the following CS-paths exist for sufficiently small $\varepsilon>0$.

Let $E_{0}$ be a CS-path from $p$ to $q$ formed by the reversible deformation of

- extending along $A_{i}$

- contracting along $A_{j}$ by $\varepsilon$, and

- extending along $A_{k}$.

Let $E_{i}$ be a CS-path from $p$ to $q$ formed by the CS-path deformation of

- introducing a straight in the direction $\alpha_{i}$,

- contracting along $A_{j}$ by $\varepsilon$, and

- extending along $A_{k}$.

Let $E_{k}$ be a CS-path from $p$ to $q$ formed by the CS-path deformation of

- extending along $A_{i}$,

- contracting along $A_{j}$ by $\varepsilon$, and

- introducing a straight in the direction $\alpha_{k}$. 
Let $E_{i k}$ be a CS-path from $p$ to $q$ formed by the CS-path deformation of

- introducing a straight in the direction $\alpha_{i}$,

- contracting along $A_{j}$ by $\varepsilon$, and

- introducing a straight in the direction $\alpha_{k}$.

These four deformations are illustrated in Figure 8 where + denotes extending if $\theta$ is displayed or introducing a straight if $\alpha$ is displayed, while - denotes contracting.
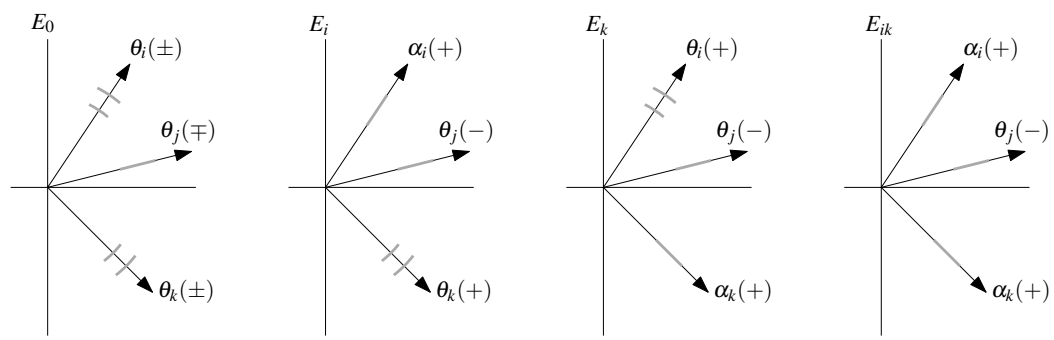

Fig. 8 Reversible deformation $E_{0}$, and irreversible deformations $E_{i}, E_{k}$ and $E_{i k}$

Let $\mathrm{d} T_{m}=T\left(E_{m}\right)-T(E)$ for $m=0, i, k, i k$. Based on the deformations, it can be seen that $\mathrm{d} T_{0}+\mathrm{d} T_{i k}=\mathrm{d} T_{i}+\mathrm{d} T_{k}$. Since the velocity points are collinear, $\mathrm{d} T_{i k}=0$. If $d T_{0}=0$, $d T_{i}=-d T_{k}$. However, since $E_{i}$ involves deformations where only 2 of the 3 deformations are straights, it follows that $d T_{i} \neq 0$ for a sufficiently small $\varepsilon$ by property (3) of Lemma 5 . Hence, either $\mathrm{d} T_{i}<0, \mathrm{~d} T_{k}<0$ or $\mathrm{d} T_{0} \neq 0$. If $\mathrm{d} T_{i}<0$ then $T\left(E_{i}\right)<T(E)$ while if $\mathrm{d} T_{k}<0$ then $T\left(E_{k}\right)<T(E)$. If $\mathrm{d} T_{0} \neq 0$ then since it is a reversible deformation, the result is shown.

Lemma 10 Let $E$ be a CS-path from $p$ to $q$ which has a pair of repeated reversible directions $A_{i}, A_{j}$ where $\rho_{i} \neq 0$. If $v$ is strict, then $E$ is non-optimal.

Proof Without loss of generality, let $\rho_{i}=1$. Since $A_{i}, A_{j}$ are repeated, $\rho_{j}=1$.

Let $E_{1}$ be a CS-path formed from $E$ by:

- contracting along $A_{i}$ by $\gamma_{i}$;

- introducing an $\mathcal{S}$ subpath of length $4 \sin \gamma_{i}$ in the direction $\theta_{i}$ in either of the 2 arcs of the $\mathcal{C C}$ subpath corresponding to $A_{j}$.

Let $E_{2}$ be a CS-path formed from $E$ by:

- contracting along $A_{i}$ by $\gamma_{i}$;

- introducing an $\mathcal{S}$ subpath of length $2 \tan \gamma_{i}$ in the direction $\alpha_{i}+2 \gamma_{i}$ in either of the $2 \operatorname{arcs}$ of the $\mathcal{C C}$ subpath corresponding to $A_{j}$

- introducing an $\mathcal{S}$ subpath of length $2 \tan \gamma_{i}$ in the direction $\alpha_{i}$ at the inflection point corresponding to $A_{j}$.

Let $q_{1}$ and $q_{2}$ denote the endpoints of the CS-paths $E_{1}$ and $E_{2}$ respectively. Clearly, $q_{1}=q_{2}=q$ by construction. Recall that $K=v^{2}+2 v^{\prime 2}-v v^{\prime \prime}$ from Definition 1 and the sign of $K$ represents the sign of the curvature of the polar function $v$. Since $v$ is continuous piecewise $C^{2}$ and strict, we can choose $\gamma_{i}>0$ sufficiently small so that either

1. $K>0$ for all $\alpha \in\left(\alpha_{i}, \alpha+2 \gamma_{i}\right)$, or

2. $K<0$ for all $\alpha \in\left(\alpha_{i}, \alpha+2 \gamma_{i}\right)$. 
If case (1) is true, then let the line tangential to $v(\alpha)$ at $\alpha=\theta_{i}$ be given by the polar function $\underline{v}(\alpha)$. Let $\underline{c}=1 / \underline{v}$. Since $K>0$ for all $\alpha \in\left(\alpha_{i}, \alpha+2 \gamma_{i}\right)$, it follows that $v(\alpha)<\underline{v}(\alpha)$ for all $\alpha \in\left(\alpha_{i}, \alpha+2 \gamma_{i}\right)$. Hence,

$$
\begin{aligned}
T\left(E_{1}\right) & =T(E)+4 \sin \gamma_{i} c\left(\theta_{i}\right)-2 \int_{-\gamma_{i}}^{\gamma_{i}} c\left(\theta_{i}+\phi\right) \mathrm{d} \phi \\
& <T(E)+4 \sin \gamma_{i} c\left(\theta_{i}\right)-2 \int_{-\gamma_{i}}^{\gamma_{i}} \underline{c}\left(\theta_{i}+\phi\right) \mathrm{d} \phi \\
& =T(E)+4 \sin \gamma_{i} \underline{c}\left(\theta_{i}\right)-2 \int_{-\gamma_{i}}^{\gamma_{i}} \underline{c}\left(\theta_{i}+\phi\right) \mathrm{d} \phi \\
& =T(E)
\end{aligned}
$$

The final step is due to the triangle inequality being satisfied by equality, since $\underline{v}(\alpha)$ is a straight line in polar coordinates.

If case (2) is true, then let the line passing through the points $\left(v\left(\alpha_{i}\right), \alpha_{i}\right)$ and $\left(v\left(\alpha_{i}+\right.\right.$ $\left.\left.2 \gamma_{i}\right), \alpha_{i}+2 \gamma_{i}\right)$ in polar coordinates be given by the polar function $\bar{v}(\alpha)$. Let $\bar{c}=1 / \bar{v}$. Since $K<0$ for all $\alpha \in\left(\alpha_{i}, \alpha+2 \gamma_{i}\right)$, it follows that $v(\alpha)<\bar{v}(\alpha)$ for all $\alpha \in\left(\alpha_{i}, \alpha+2 \gamma_{i}\right)$. Hence,

$$
\begin{aligned}
T\left(E_{2}\right) & =T(E)+2 \tan \gamma_{i}\left(c\left(\alpha_{i}\right)+c\left(\alpha_{i}+2 \gamma_{i}\right)\right)-2 \int_{-\gamma_{i}}^{\gamma_{i}} c\left(\theta_{i}+\phi\right) \mathrm{d} \phi \\
& <T(E)+2 \tan \gamma_{i}\left(\bar{c}\left(\alpha_{i}\right)+2 \bar{c}\left(\alpha_{i}+2 \gamma_{i}\right)\right)-2 \int_{-\gamma_{i}}^{\gamma_{i}} \bar{c}\left(\theta_{i}+\phi\right) \mathrm{d} \phi \\
& =T(E)
\end{aligned}
$$

Hence, $E$ is non-optimal in both cases.

\section{Definition 5 In a CS-path,}

- an inserted $\mathcal{S}$ segment, denoted $\widetilde{\mathcal{S}}$, is an $\mathcal{S}$ segment preceded and followed by $\mathcal{C}$ arcs of opposing sense

- a linked $\mathcal{S}$ segment, denoted $\breve{\mathcal{S}}$, is an $\mathcal{S}$ segment preceded and followed by $\mathcal{C}$ arcs of the same sense

- a proper linked $\mathcal{S}$ segment, denoted $\underline{\mathcal{S}}$, is an $\breve{\mathcal{S}}$ segment where the preceding and following arcs are of combined length less than $2 \pi$

- an improper linked $\mathcal{S}$ segment, denoted $\overline{\mathcal{S}}$, is an $\breve{\mathcal{S}}$ segment that is not an $\underline{\mathcal{S}}$ segment

Lemma 11 Let $E$ be a CS-path from $p$ to $q$ such that:

$-d \geq 4$

- no 3 reversible directions of $E$ are distinct.

If $v$ is strict, then $E$ is non-optimal.

Proof Note that in any CS-path, $\alpha_{i} \neq \alpha_{i+1} \forall i<d$. Hence, for the path $E, A_{m}=A_{l}$ for $m, l$ odd, while $A_{m}=A_{l}$ for $m, l$ even, and $\alpha_{1} \neq \alpha_{2}$. If $\rho_{1} \neq 0$ or $\rho_{2} \neq 0$, the path is non-optimal by Lemma 10 . Hence we simply need to consider the case of $\rho_{m}=0$ for all $m \in\{1, \cdots, d\}$. 
If $A_{2}$ corresponds to an $\breve{\mathcal{S}}$, the combined lengths of the arcs preceding and following this straight must be of length $2 \pi$ because $A_{1}=A_{3}$. Since $d \geq 4$, it is possible to contract along $A_{2}$ until it degenerates while extending along $A_{4}$ by an equal amount. This would result in a new path $E^{\prime}$ from $p$ to $q$ where $T\left(E^{\prime}\right)=T(E)$. However, $E^{\prime}$ has an arc of length $2 \pi$ and hence both $E^{\prime}$ and $E$ are non-optimal.

If $A_{2}$ corresponds to an $\widetilde{\mathcal{S}}$, contracting along $A_{2}$ until it degenerates while extending along $A_{4}$ by an equal amount would result in a $\mathcal{C C}$ subpath being formed where $A_{2}$ has degenerated to a point of inflection, which has a new reversible direction distinct to $A_{1}$ and $A_{4}$ since $\rho_{2} \neq 0$. The result then follows from Lemma 9 .

Lemma 12 Let $E$ be a CS-path from $p$ to $q$ which has a pair of repeated reversible directions $A_{i}, A_{j}$ such that:

$-\rho_{i}=0$

- at least one of $A_{i}, A_{j}$ corresponds to an $\widetilde{\mathcal{S}}$ or $\overline{\mathcal{S}}$.

If $v$ is strict, then $E$ is non-optimal.

Proof Clearly, there must exist a reversible direction $A_{k}$ which is distinct from $A_{j}$ for such a path to exist. Without loss of generality, let $A_{i}$ correspond an $\widetilde{\mathcal{S}}$ or $\overline{\mathcal{S}}$. By contracting the straight edge in each of these 2 cases, the result follows in a similar manner to the proof of Lemma 11.

Lemma 13 Let $E$ be a CS-path such that:

- E has at least 3 distinct reversible directions;

- all the reversible directions of $E$ lie in an open half-space of $\mathbb{R}^{2}$;

- at least one of the interior reversible directions of $E$ corresponds to an $\widetilde{\mathcal{S}}$ or $\overline{\mathcal{S}}$.

If $v$ is strict, then $E$ is non-optimal.

Proof From Lemmas 8 and 9, only the case where the velocity points of all the reversible directions are all collinear, and $\rho_{1}, \cdots, \rho_{d}=0$ needs to be considered. Let $A_{i}, A_{k}$ be the exterior reversible directions, and $A_{j}$ be an interior direction corresponding to an $\widetilde{\mathcal{S}}$ or $\overline{\mathcal{S}}$.

Since $\rho_{i}, \rho_{j}, \rho_{k}=0, A_{j}$ can be contracted until it degenerates, while $A_{i}$ and $A_{k}$ are extended to satisfy (1). The new path formed $E^{\prime}$ has $q^{\prime}=q$ and $T\left(E^{\prime}\right)=T(E)$ since the velocity points are collinear, but in a similar manner to the proof of Lemma $11, E^{\prime}$ and $E$ can be shown to be non-optimal by considering the 2 cases of $A_{j}$ corresponding to an $\widetilde{\mathcal{S}}$ or $\overline{\mathcal{S}}$.

4.2 Reducible path forms

Lemma 14 Let $E$ be a CS-path from $p$ to $q$ which has a pair of repeated reversible directions $A_{i}, A_{j}$ such that:

- $\rho_{i}=0$;

- at least one of $A_{i}, A_{j}$ corresponds to an $\underline{\mathcal{S}}$ first label, or last label of the CS-path.

Then $E$ is reducible.

Proof Without loss of generality, let $A_{i}$ correspond to an $\underline{\mathcal{S}}$, first label, or last label of the CS-path. Contracting along $A_{i}$ until it degenerates while extending along $A_{j}$ by an equal amount results in a new path $E^{\prime}$ with $q^{\prime}=q$ where $T\left(E^{\prime}\right)=T(E)$ but $E^{\prime}$ is of degree 1 lower than $E$. 
Lemma 15 Let $E$ be a CS-path such that:

- it has at least 3 distinct reversible directions;

- all its reversible directions lie in an open half-space of $\mathbb{R}^{2}$;

$-\rho_{1}, \cdots, \rho_{d}=0$

- at least one of its interior reversible directions corresponds to an $\underline{\mathcal{S}}$, first label, or last label of the CS-path.

- the velocity points of all of its reversible directions are collinear.

Then $E$ is reducible.

Proof Let $A_{i}, A_{k}$ be the exterior reversible directions, and $A_{j}$ be an interior direction corresponding to an $\underline{\mathcal{S}}$, first label, or last label of the CS-path. Since $\rho_{i}, \rho_{j}, \rho_{k}=0, A_{j}$ can be contracted until it degenerates, while $A_{i}$ and $A_{k}$ are extended to satisfy (1). The new path formed $E^{\prime}$ has $q^{\prime}=q$ where $T\left(E^{\prime}\right)=T(E)$ but $E^{\prime}$ is of degree 1 lower than $E$.

\subsection{Main result}

Let $\Pi$ denote a special case of a $\mathcal{C}$ arc which is of length $\pi$.

Definition 6 Let the sufficient path set $\mathbb{D}_{p q}^{S}$ be the set of all CS-paths from $p$ to $q$ each of which has degree $d \leq 2$ which do not have an $\mathcal{S} \Pi \mathcal{S}$ subpath. This is equivalent to defining $\mathbb{D}_{p q}^{S}$ as the set of all $\mathcal{C} \mathcal{S C S C}$ paths and degeneracies, which do not have an $\mathcal{S} \Pi \mathcal{S}$ subpath.

Let the necessary path set $\mathbb{D}_{p q}^{N}$ be the set of all CS-paths from $p$ to $q$ each of which either

1. belong to $\mathbb{D}_{p q}^{S}$;

2. have $d=3, \alpha_{1}=\alpha_{3}, \rho_{1}=\rho_{3}=0$, where both $A_{1}$ and $A_{3}$ correspond to an $\underline{\mathcal{S}}$, first label, or last label of the CS-path, and $A_{2} \neq\left(\alpha_{1}+\pi, 0\right)$;

3. have $d \geq 3$, at least 3 distinct reversible directions, $\rho_{1}, \cdots, \rho_{d}=0$ and $A_{1}, \cdots, A_{d}$ lie strictly in a half-space of $\mathbb{R}^{2}$, with each interior reversible direction corresponding to an $\underline{\mathcal{S}}$, first label, or last label of the CS-path.

Let $\mathbb{D}_{p q}$ denote the set of all Dubins paths from $p$ to $q$. Let $\mathbb{P}_{p q}^{C S}$ denote the set of all CS-paths from $p$ to $q$. Note that by Definition $6, \mathbb{D}_{p q} \subset \mathbb{D}_{p q}^{S} \subset \mathbb{D}_{p q}^{N} \subset \mathbb{P}_{p q}^{C S} \subset \mathbb{P}_{p q}$.

Theorem 2 If $v$ is strict, every optimal curvature-constrained path from $p$ to $q$ belongs to $\mathbb{D}_{p q}^{N}$. Furthermore, there exists a path in $\mathbb{D}_{p q}^{S}$ which is optimal.

Proof It follows from Lemmas 6 - 13 that every optimal curvature-constrained path from $p$ to $q$ belongs to $\mathbb{D}_{p q}^{N}$. Applying Lemmas 8, 14 and 15 to this result tells us that any optimal path of types (2) and (3) in $\mathbb{D}_{p q}^{N}$ is reducible to a path belonging to $\mathbb{D}_{p q}^{S}$. Hence, there exists a path in $\mathbb{D}_{p q}^{S}$ which is optimal.

Corollary 3 Given any velocity function $v$, there exists a path of the form $\mathcal{C S C S C}$ (or degeneracy) which is an optimal curvature-constrained path from $p$ to $q$.

Proof If $v$ is strict, the result follows from Theorem 2 . Hence, only weak (not strict) velocity functions need to be considered.

Let $v$ be a weak velocity function, where $c=1 / v$. Let $\left\{I_{j}\right\}$ be the set of all maximal open intervals $I_{j}=\left(\alpha_{j}-\beta_{j}, \alpha_{j}+\beta_{j}\right)$, where $K(\alpha)=0 \forall \alpha \in I_{j}, \forall j$ (and each $\beta_{j}$ is as large as possible). Let $h_{j}(\alpha)$ be defined as follows: 


$$
h_{j}(\alpha)=\left\{\begin{array}{cc}
\beta_{j}-\left|\alpha-\alpha_{j}\right| & \text { if } \alpha \in I_{j} \\
0 & \text { otherwise }
\end{array}\right.
$$

Consider a new velocity function $v_{\varepsilon}=1 / c_{\varepsilon}$ where $c_{\varepsilon}=c+\varepsilon \sum_{j} h_{j}(\alpha)$. By construction, $v_{\varepsilon}$ is strict for any $\varepsilon>0$. Let $T(E)$ and $T_{\mathcal{\varepsilon}}(E)$ denote the costs of path $E$ with respect to the directional-cost functions $c$ and $c_{\varepsilon}$ respectively. Let $H(E)=\int_{E} \sum_{j} h_{j}(\alpha) \mathrm{d} s$.

Suppose $\exists E \in \mathbb{P}_{p q} \backslash \mathbb{D}_{p q}^{N}$ such that $T(E)<T(D) \forall D \in \mathbb{D}_{p q}^{N}$. Then,

$$
\begin{aligned}
T_{\mathcal{\varepsilon}}(E) & =T(E)+\varepsilon H(E) \\
& <T(D), \text { by choosing } \varepsilon \in(0,(T(D)-T(E)) /(H(E))) \\
& <T_{\varepsilon}(D), \forall D \in \mathbb{D}_{p q}^{N}
\end{aligned}
$$

However, Theorem 2 states that there cannot exist such a $E$ since $v_{\varepsilon}$ is strict and $E \notin \mathbb{D}_{p q}^{N}$. Hence, by contradiction, there cannot exist a path in $\mathbb{P}_{p q} \backslash \mathbb{D}_{p q}^{N}$ which is of less cost than all the paths in $D \in \mathbb{D}_{p q}^{N}$. Finally, by applying Lemmas 8,14 and 15 , we get the result.

4.4 Extension - the weighted arcs problem

The motivation of this extension is related to the underground mine development application, where curved sections may require additional support and hence incur a higher development cost. There are also additional ventilation costs due to shock losses around bends [7]. In this extension, we model these effects by imposing a scaling factor $w^{C} \geq 1$ on $\mathcal{C}$ subpaths, as well as having a directional-cost. This problem is only well-posed if we restrict ourselves to considering only CS-paths.

Definition 7 Let $E$ be a CS-path from $p$ to $q$, which is made up of subpaths $E_{1}, \cdots, E_{n}$, corresponding to its $n$ labels.

$$
T^{C}(E)=\sum_{i=1}^{n} w_{i} T\left(E_{i}\right), \text { where } w_{i}=\left\{\begin{aligned}
w^{C} & \text { if } E_{i} \text { is a } \mathcal{C} \text { arc } \\
1 & \text { if } E_{i} \text { is an } \mathcal{S} \text { straight }
\end{aligned}\right.
$$

A path $E$ is an optimal weighted-arc CS-path from $p$ to $q$ if $E \in\left\{P \in \mathbb{P}_{p q}^{C S}: T^{C}(P) \leq\right.$ $\left.T^{C}(Q) \forall Q \in \mathbb{P}_{p q}^{C S}\right\}$

Corollary 4 Given any velocity function $v$, there exists a path in $\mathbb{D}_{p q}^{S}$ which is an optimal weighted-arc CS-path from $p$ to $q$. Furthermore, if $v$ is strict, every optimal weighted-arc CS-path belongs to $\mathbb{D}_{p q}^{N}$.

Proof The equivalent results to Lemmas 6 - 8 and 14 hold without any modification to the proofs. Lemmas 9 and 15 hold by simply replacing velocity point with equivalent velocity point defined as follows. The equivalent velocity point of a reversible direction $A_{i}$ is the point in $\mathbb{R}^{2}$ which in polar coordinates is:

- $\left(w^{C} v\left(\alpha_{i}\right), \alpha_{i}\right)$ if $\rho_{i}=0$

- $\left(v\left(\alpha_{i}\right), \alpha_{i}\right)$ if $\rho_{i} \neq 0$. 
Lemma 10 applies because of the condition that $w^{C} \geq 1$. Lemmas $11-13$ then hold as their arguments make use of the preceding lemmas. Finally, similar proofs to Theorem 2 and Corollary 3 hold by replacing $\mathbb{P}_{p q}$ with $\mathbb{P}_{p q}^{C S}$.

Remark 1 It is clear that since Lemma 10 only holds when $w^{C} \geq 1$, the problem is not wellposed if we consider $w^{C}<1$. This is because any straight subpath $E_{i}$ could be replaced by a sequence of infinitesimally short arcs approximating $E_{i}$, which in the limit, $\operatorname{cost}{ }^{C} T\left(E_{i}\right)$.

\section{Conclusion}

This paper shows that given any velocity function and pair of directed points, there exists a path of the form $\mathcal{C S C S C}$ or a degeneracy which is optimal. Furthermore, if we impose a scaling factor $w^{C} \geq 1$ on the cost of $\mathcal{C}$ subpaths, this result still holds. This result tells us that if we wish to construct an optimal path based on a given velocity function, $w^{C} \geq 1$, together with starting and end points, we need only consider a small subset of the entire set of curvature-constrained paths. The next step is to use the ideas developed in this paper to come up with necessary conditions for optimality of a given path in relation to the velocity function, and design an algorithm which can efficiently construct optimal paths. This further work will appear in a forthcoming paper.

Acknowledgements This research is supported by a grant from the Australian Research Council

\section{References}

1. J.D. Boissonnat, A. Cerezo, and J. Leblond, Shortest paths of bounded curvature in the plane, J. Intell. Robot. Syst. 11 (1994), 5-20.

2. M. Brazil, P.A. Grossman, D.H. Lee, J.H. Rubinstein, D.A. Thomas, and N.C. Wormald, Decline design in underground mines using constrained path optimisation, Min. Technol.: Trans. Inst. Min. Metall., Sect. A 117 (2008), no. 2, 93-99.

3. A.J. Chang, M. Brazil, J.H. Rubinstein, and D.A. Thomas, Curvature-constrained directional-cost paths in the plane, J. Glob. Optim. (2011), 1-19, 10.1007/s10898-011-9730-1.

4. I.S. Dolinskaya, Optimal path finding in direction, location and time dependent environments, $\mathrm{Ph} . \mathrm{D}$. thesis, Industrial and Operations Engineering, The University of Michigan, 2009.

5. L.E. Dubins, On curves of minimal length with a constraint on average curvature, and with prescribed initial and terminal positions and tangents, Am. J. Math. 79 (1957), 497-516.

6. D.H. Laubscher, A geomechanics classification system for the rating of rock mass in mine design, J. S. Atr. Inst. Min. Metal 90 (1990), no. 10, 257-273.

7. M.J. McPherson, Subsurface ventilation and environmental engineering, Chapman \& Hall, 1993.

8. P. Soueres and J. Boissonnat, Optimal trajectories for nonholonomic mobile robots, Robot Motion Planning and Control (J. Laumond, ed.), Lecture Notes in Control and Information Sciences, vol. 229, Springer Berlin / Heidelberg, 1998, 10.1007/BFb0036072, pp. 93-170.

9. P. Widmayer, Y.F. Wu, and C.K. Wong, On some distance problems in fixed orientations, SIAM J. Comput. 16 (1987), no. 4, 728-746. 


\section{University Library}

\section{- M M N E R VA A gateway to Melbourne's research publications}

Minerva Access is the Institutional Repository of The University of Melbourne

Author/s:

Chang, AJ;Brazil, M;Rubinstein, JH;Thomas, DA

Title:

Optimal curvature-constrained paths for general directional-cost functions

Date:

2013-09-01

Citation:

Chang, A. J., Brazil, M., Rubinstein, J. H. \& Thomas, D. A. (2013). Optimal curvatureconstrained paths for general directional-cost functions. OPTIMIZATION AND ENGINEERING, 14 (3), pp.395-416. https://doi.org/10.1007/s11081-011-9180-0.

Persistent Link:

http://hdl.handle.net/11343/282834 